\title{
The judgement of the eye
}

\author{
Timothy F. Christian, MD, MPA ${ }^{\text {a }}$ \\ ${ }^{a}$ Rutgers University, New Brunswick, NJ \\ Received Jan 8, 2016; accepted Jan 8, 2016 \\ doi: $10.1007 / \mathrm{s} 12350-016-0412-8$
}

\section{BEAUTY IS BOUGHT BY JUDGEMENT OF THE EYE ${ }^{1}$}

The point-counterpoint of Saab et al, and Reynes et $\mathrm{al}^{2,3}$ is very well done and reminiscent of the Mirror of Erised of the Harry Potter series in which one sees their desire while gazing into it. Both make compelling arguments on the impact of caffeine ingestion on the accuracy of SPECT myocardial perfusion imaging. Both quote from the same studies, yet see opposite but credible conclusions. Clearly, there is a literature supporting an inhibitory action of caffeine-containing products on coronary vasodilation mediated by adenosine A2 receptors but the timing, dosage, vasodilator agent, and specific genetic predisposition to caffeine variability clouds the looking glass. The first description of caffeine impact on dipyridamole-mediated vasodilation was in $1989 .{ }^{4}$ Twenty-five years later, the impact of the issue is still uncertain. Why are we not further along? This piece will outline some concepts that have contributed to the confusion. These are within the categories of imaging algorithms, image displays, physics, tracer kinetics, and statistics.

\section{TEMPORAL REPRODUCIBILITY}

Most of the small studies exploring the impact of caffeine on vasodilation rely on serial perfusion imaging studies. While inter- and intra-observer variability for interpreting SPECT perfusion studies has been fairly well defined, ${ }^{5}$ temporal reproducibility has not. Despite common clinical utilization of prior perfusion studies to detect changes in the coronary circulation over time in patients, there are limited data on the reproducibility of such comparisons by SPECT imaging. ${ }^{6}$ Consequently, it is hard to interpret vasodilation perfusion studies of

\footnotetext{
Reprint requests: Timothy F Christian, MD, MPA, Rutgers University,

New Brunswick, NJ; timothy.christian@rutgers.edu

J Nucl Cardiol 2016;23:454-6.

$1071-3581 / \$ 34.00$

Copyright (c) 2016 American Society of Nuclear Cardiology.
}

small sample size, performed with and without caffeine on board, as there is not a clear quantitative threshold by SPECT imaging that constitutes a significant change. If there was a standard quantitative methodology, the confidence in determining a serial change between studies would be greater.

\section{MYOCARDIAL MODELS OF MYOCARDIAL PERFUSION VARY}

Another confounder is the use of segmented myocardial perfusion models. These truncate the ability to detect changes that are spread over two segments, diluting the difference. This can be a limitation. Another is the use of the summed stress and rest scores which tabulates all hypo-perfused segments on the images. The 17-segment perfusion model is the clinical approach that is relevant due to its widespread adoption despite shortcomings. While the summed stress score is widely used for clinical interpretation of images, a disadvantage is the mixing of reversible viable segments with fixed nonviable segments. This method is present in a number of the studies cited by the authors. If caffeine has a clinical impact on perfusion images, it will be found only in the viable segments. Nonviable segments will not take up conventional SPECT perfusion agents despite the presence of blood flow. The summed stress score mixes these together. Limiting the analysis to the reversibility score avoids the confusion. Several studies referenced have used a measure of defect size as a continuous variable (area of the LV) which should be able to detect more subtle changes. This is a better approach but, as we will see, the relative nature of perfusion imaging can make any serial image comparison problematic.

\section{ABSOLUTE VS RELATIVE MYOCARDIAL BLOOD FLOW}

Another confounding factor is the use of relative myocardial blood flow (MBF) algorithms for image reconstruction. Three small studies have shown a 


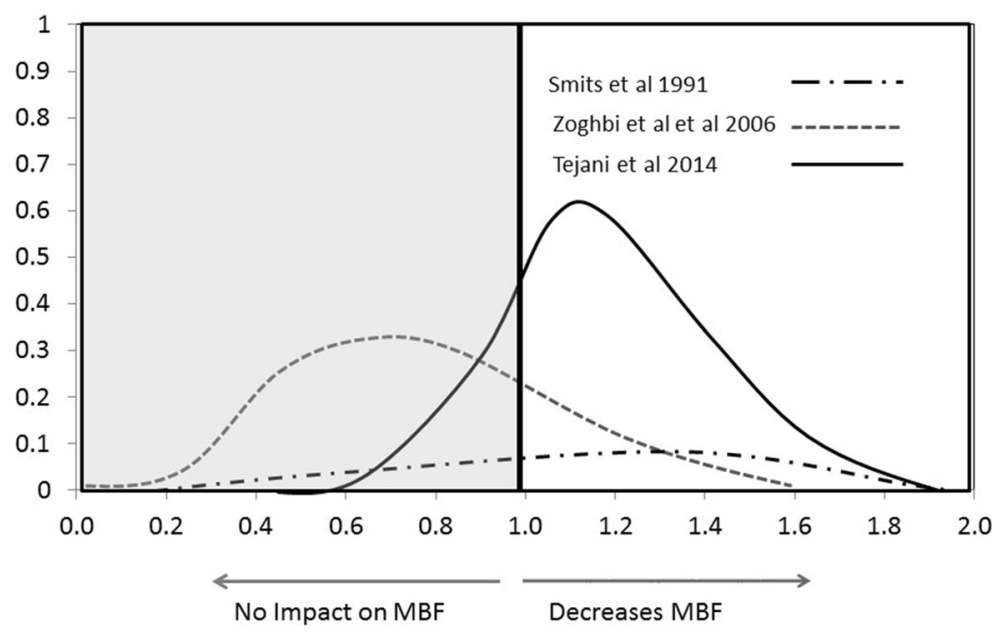

Figure 1. Potential probability curves estimating the impact of caffeine ingestion on vasodilation perfusion SPECT imaging from three papers cited by the discussants. ${ }^{11,13,14}$ The height of the curve is a reflection of the sample size with the peak corresponding to the mean probability estimate of the impact of caffeine on SPECT clinical imaging, and the width reflecting the confidence interval of the prediction. Each curve is cumulative from the previous such that the final curve includes probability estimates from that study plus the estimate of the impact of the two prior studies combined. These curves are hypothetical only and not accurate reflections of a Bayesian analysis.

decline in absolute MBF (least pronounced when regadenoson was used as the vasodilating agent) in the presence of caffeine as assessed by PET perfusion imaging. ${ }^{7-9}$ But this does not necessarily translate to a change in perfusion imaging using SPECT techniques. Because of the lack of an arterial input function for most protocols and significant attenuation and scatter, SPECT imaging is not amenable to quantifying absolute MBF. Alternatively, a relative signal intensity algorithm is necessary to compare images by bringing up the maximum signal intensity to maximal brightness within each image. This region can vary between studies and limit the ability to recognize a decrease in vasodilation as the serial images are of equal signal intensity in at least one zone. There is no reason not to assume that stress flow in normal and abnormal flow reserve zones will not be reduced equally, thus limiting the ability to detect a decrease in vasodilation by caffeine. Consequently, we are left deducing whether caffeine is impacting vasodilation by a decrease in defect reversibility in zones supplied by diseased arteries rather than by simply measuring $\mathrm{MBF}$ in the myocardium.

\section{ROLL-OFF UPTAKE KINETICS OF SPECT PERFUSION AGENTS}

Early work on both Tc-99m-isonitrile tracers and Thallium-201 demonstrates that uptake of these perfusion tracers is linear at lower flow rates but plateaus at
MBF values of 2.0-2.5 ml/minute/g. ${ }^{10}$ Vasodilator stress increases $\mathrm{MBF}$ to $4.0-5.0 \mathrm{ml} / \mathrm{minute} / \mathrm{g}$ in normally perfused myocardium. No SPECT tracer can reflect that level of MBF. The three absolute MBF studies quoted in the pro and con articles ${ }^{7-9}$ show MBF vasodilation values without caffeine ranging from 2.97 to $4.11 \mathrm{ml} /$ minute/g and with caffeine of $2.25-2.75 \mathrm{ml} /$ minute $/ \mathrm{g}$. All of these values are at or higher than the uptake plateau flow rate. Consequently, it may not be possible to consistently show an impact of caffeine on MBF vasodilation using SPECT tracers.

\section{FREQUENTIST VS BAYESIAN STATISTICS}

The approach of both discussants regarding the clinical literature is to treat each study independently. Consequently, there is no relationship between prior studies and no common thread to tie them together. In such an approach, sample size becomes important and randomized trials (of which there is one ${ }^{11}$ ) become critical. This is known as a frequentist approach to scientific analysis. I would argue that this approach does not work here because of the small sample sizes and variability in design between studies. Rather a Bayesian approach would provide more clarity.

In a frequentist approach, any knowledge or evidence available prior to the measurement period is not used to generate conclusions, although it may be incorporated into the study design. In addition, the required sample size for the control and treatment 
groups needs to provide a result that will stand on its own statistically; a treatment will either be deemed effective at a statistically significant level, or it will not.

In contrast, the Bayesian approach does use prior knowledge or evidence to generate conclusions. The theorem asks, "What is the probability that a new result is providing a true estimate of the effect in question given the evidence accumulated to this point?' In other words, the likelihood of getting a particular result from a clinical trial, formally known as the posterior probability, depends on the findings of the trial itself as well as the pre-trial probability of the results of all the previous studies to date, formally called the prior probability distribution. Each study provides an estimate of the true impact of caffeine on MBF with a confidence interval for that prediction that can be analyzed as a cumulative variable. $^{12}$ We could order these chronologically and establish a prior probability after each study is added, so as to derive the cumulative probability estimate based on all the data. Studies can be weighted by sample size and strength of design. A hypothetical graphic of that approach is shown in Figure 1. ${ }^{11,13,14}$

Bayesian analysis places the accumulated data in perspective with a final estimate of the impact of caffeine on $\mathrm{MBF}$ with a confidence interval of that estimate. This could be done with the studies cited in the arguments for the impact of caffeine ingestion prior to vasodilation stress. In the absence of that, looking at the data using an eyeball approach, it looks like the confidence interval regarding the impact of caffeine still crosses 1.0 but is leaning toward the impact side. But leaving it to the eye allows beauty to be judged subjectively. A large randomized trial could settle the issue using both absolute MBF with PET and serial SPECT imaging with a defined reproducibility threshold that must be eclipsed for significance and a standardized protocol. But we should not lose sight of the good prognosis associated with a normal myocardial perfusion study, most recently confirmed with regadenoson vasodilation. ${ }^{15}$ Events are rare. Consequently, it is not likely that there is a large cohort of coffee drinkers with falsely negative vasodilator perfusion studies out there. A better approach might be to direct effort and resources into lower cost laboratory quality metrics aimed at preventing patients from ingesting caffeine prior to testing. Then the eye can focus on the judgement of the images.

\section{References}

1. Shakespeare W. Loves labours lost. Act 2, Scene 1.

2. Saab R, Bajaj NS, Hage FG. Caffeine does not significantly reduce the sensitivity of vasodilator stress myocardial perfusion imaging. J Nucl Cardiol 2016. doi:10.1007/s12350-015-0364-4.

3. Reyes E. Caffeine reduces the sensitivity of vasodilator MPI for the detection of myocardial ischaemia: Pro. J Nucl Cardiol 2016. doi:10.1007/s12350-015-0371-5.

4. Smits P, Aengevaeren WR, Corstens FH, Thien T. Caffeine reduces dipyridamole-induced myocardial ischemia. $\mathrm{J}$ Nucl Med 1989;30:1723-6.

5. Johansen A, Gaster AL, Veje A, Thayssen P, Haghfelt T, HølundCarlsen PF. Interpretive intra- and interobserver reproducibility of rest/stress 99Tcm-sestamibi myocardial perfusion SPECT in a consecutive group of male patients with stable angina pectoris before and after percutaneous transluminal angioplasty. Nucl Med Commun 2001;22:531-7.

6. Iskandrian AE, Hage F, Shaw LJ, Mahmarian JJ, Berman DS. Serial myocardial perfusion imaging: Defining a significant change and targeting management decisions. JACC Cardiovasc Imaging 2014;7:79-96.

7. Gaemperli O, Schepis T, Koepfli P, Siegrist PT, Fleischman S, Nguyen $P$, et al. Interaction of caffeine with regadenoson-induced hyperemic myocardial blood flow as measured by positron emission tomography: A randomized, double-blind, placebo-controlled crossover trial. J Am Coll Cardiol 2008;51:328-9.

8. Bottcher M, Czernin J, Sun KT, Phelps ME, Schelbert HR. Effect of caffeine on myocardial blood flow at rest and during pharmacological vasodilation. J Nucl Med 1995;36:2016-21.

9. Kubo S, Tadamura E, Toyoda H, Mamede M, Yamamuro M, Magata Y, et al. Effect of caffeine intake on myocardial hyperemic flow induced by adenosine triphosphate and dipyridamole. J Nucl Med 2004;45:730-8.

10. Glover DK, Okada RD. Myocardial kinetics of Tc-MIBI in canine myocardium after dipyridamole. Circulation 1990;81:628-36.

11. Tejani FH, Thompson RC, Kristy R, Bukofzer S. Effect of caffeine on SPECT myocardial perfusion imaging during regadenoson pharmacologic stress: A prospective randomized multicenter study. Int J Cardiovasc Imaging 2014;30:979-89.

12. Spiegelhalter DJ, Myles JP, Jones DR, Abrams KR. Bayesian methods in health technology assessment: A review. Health Technol Assess 2000; 4.

13. Smits P, Corstens FH, Aengevaeren WR, Wackers FJ, Thien T. False-negative dipyridamole-thallium 201 myocardial imaging after caffeine infusion. J Nucl Med 1991;32:1538-41.

14. Zoghbi GJ, Aqel R, Blackmon L, Heo J, Iskandrian AE. Effect of caffeine on ischemia detection by adenosine single photon emission computed tomography perfusion imaging. J Am Coll Cardiol 2008;52:2008-16

15. Iqbal FM, Hage FG, Ahmed A, Dean PJ, Raslan S, Heo J, et al. Comparison of the prognostic value of normal regadenoson with normal adenosine myocardial perfusion imaging with propensity score matching. JACC Cardiovasc Imaging 2012;5:1014-21. doi: 10.1016/j.jcmg.2012.04.009. 